\title{
Ascertainment of cause-specific mortality in COPD: operations of the TORCH Clinical Endpoint Committee
}

\author{
Lorcan P McGarvey, Matthias John, Julie A Anderson, Michael Zvarich, Robert A Wise
}

Thorax 2007;62:411-415. doi: 10.1136/thx.2006.072348

See end of article for authors' affiliations

Correspondence to:

Dr Lorcan P McGarvey, The

Queen's University of

Belfast, Grosvenor Road,

Belfast BT12 6BJ, UK;

l.mcgarvey@qub.ac.uk

Received 26 September 2006

Accepted 21 January 2007

Published Online First

20 February 2007

......................
Background: TORCH (Towards a Revolution in COPD Health) is an international multicentre, randomised, placebo-controlled clinical trial of inhaled fluticasone propionate/salmeterol combination treatment and its monotherapy components for maintenance treatment of moderately to severely impaired patients with chronic obstructive pulmonary disease (COPD). The primary outcome is all-cause mortality. Cause-specific mortality and deaths related to COPD are additional outcome measures, but systematic methods for ascertainment of these outcomes have not previously been described.

Methods: A Clinical Endpoint Committee (CEC) was tasked with categorising the cause of death and the relationship of deaths to COPD in a systematic, unbiased and independent manner. The key elements of the operation of the committee were the use of predefined principles of operation and definitions of cause of death and COPD-relatedness; the independent review of cases by all members with development of a consensus opinion; and a substantial infrastructure to collect medical information.

Results: 911 deaths were reviewed and consensus was reached in all. Cause-specific mortality was: cardiovascular $27 \%$, respiratory $35 \%$, cancer $21 \%$, other $10 \%$ and unknown $8 \% .40 \%$ of deaths were definitely or probably related to COPD. Adjudications were identical in $83 \%$ of blindly re-adjudicated cases $(\kappa=0.80)$. COPD-relatedness was reproduced $84 \%$ of the time $(\kappa=0.73)$. The CEC adjudication was equivalent to the primary cause of death recorded by the site investigator in $52 \%$ of cases.

Conclusion: A CEC can provide standardised, reliable and informative adjudication of COPD mortality that provides information which frequently differs from data collected from assessment by site investigators.

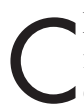
hronic obstructive pulmonary disease (COPD) is the fourth leading cause of death in the United States. ${ }^{12}$ Globally, COPD is projected to rise from the sixth leading cause of death in 1990 to the third most common cause of death in 2020. ${ }^{3}$ Clinical trials of COPD maintenance treatment have typically used lung function as a primary outcome measure, with secondary outcomes of exacerbation frequency, symptoms and quality of life. Mortality and cause-specific mortality have infrequently been used as a primary outcome measure because of the requirements for long duration of follow-up and large numbers of participants. Since the domiciliary oxygen trials done in the $1970 \mathrm{~s},{ }^{45}$ the TORCH (Towards a Revolution in COPD Health) trial was the first international trial of COPD maintenance therapy that used allcause mortality as a primary outcome measure and causespecific mortality as a secondary outcome measure. ${ }^{6}$ Moreover, because COPD may substantially contribute to mortality in the presence of other primary illnesses, COPD-related mortality was also used as an outcome measure.

Previous clinical trials of COPD have used independent review committees to assign cause of death, but the methods, operations and performance of these committees have not been published. ${ }^{78}$ Attribution of cause of death is particularly difficult for patients with COPD because they often have other comorbidities or the contribution of COPD is not taken into account. TORCH provided an opportunity to develop and evaluate methods for adjudicating causes of mortality in these patients. In TORCH, the cause of death was adjudicated by a three-member Clinical Endpoint Committee (CEC), independent of the main Steering Committee. This paper describes the operations and experience of this committee in adjudicating 911 deaths. We present results about the reliability of CEC adjudications and compare CEC cause-specific mortality with causes of death determined by local site investigators.

\section{METHODS}

\section{TORCH study design}

The TORCH trial design has been published previously. ${ }^{6}$ Briefly, the trial was a randomised, double blind, parallel group, controlled clinical trial comparing inhaled salmeterol $50 \mu \mathrm{g}$ twice daily, inhaled fluticasone propionate $500 \mu \mathrm{g}$ twice daily, combined salmeterol $50 \mu \mathrm{g}$ and fluticasone propionate $500 \mu \mathrm{g}$ inhaled twice daily and placebo. The enrolled participants had moderate to severe COPD (prebronchodilator forced expiratory volume in $1 \mathrm{~s}<60 \%$ predicted). Each participant was followed for 3 years. The primary outcome measure is all-cause mortality. Secondary outcomes include exacerbations and quality of life. Other outcome measures include lung function, cause-specific mortality and deaths related to COPD. The study enrolled 6184 participants who were assigned to treatment at 444 centres in 42 countries. Overall, 911 deaths that occurred in randomised participants were reviewed by the CEC. Of these, 875 occurred within 3 years of randomisation from nonexcluded sites and were used for analysis of the primary trial outcome. Of the 36 deaths not included in the primary efficacy analysis, 7 occurred at sites that were administratively excluded from the analysis before unmasking the data and 29 occurred more than 1092 days after randomisation. This report analyses the experience from all 911 adjudicated deaths.

\section{Acquisition of medical information}

When one of the participants died, the study site completed a serious adverse event report which was forwarded to the regional study coordinating centre within $24 \mathrm{~h}$. The site coordinator and investigator provided whatever information

Abbreviations: CEC, Clinical Endpoint Committee; COPD, chronic obstructive pulmonary disease; TORCH, Towards a Revolution in COPD Health 
was known about the cause of death at that time. Each site then conducted an investigation into the cause of the death using the death certificate, medical records including emergency department and hospital records, $x$ ray reports, laboratory reports, operative and procedure reports, histological reports from biopsy specimens and necropsy reports. In deaths that occurred in a non-medical facility, the site attempted to obtain witness interviews to describe the circumstances of the death, when the participant was last known to be alive and whether symptoms were known to precede the death. In some circumstances the sites provided additional information such as newspaper accounts, emergency services reports or medical examiner determinations. These records were reviewed for completeness at the local, regional and central study offices, and additional information was sought and acquired if possible. The site investigator was asked to provide a primary cause of death as well as secondary causes. If needed, the medical records were translated into English. The medical information was collated into files with information from the case report form including demographic and anthropometric data, serial lung function tests and medication lists.

\section{Operation of the Clinical Endpoint Committee (CEC)}

The CEC was composed of three physicians who each had clinical and research expertise in internal medicine and pulmonary and critical care medicine (LMcG, MJ, RW). The committee members represented three of the participating countries (UK, Germany and USA) but were not site investigators. The committee members and chairman were appointed by the sponsor and the composition of the committee was approved by the TORCH Steering Committee. The sponsor drafted a charter for the committee that was approved by the CEC and the TORCH Steering Committee.

The CEC had an initial organisational meeting with representatives of the study sponsor to review, modify and approve the charter, and to design relevant data collection forms. The CEC developed a series of hypothetical clinical scenarios representing complex or problematic cases such as might be reviewed by the committee. These cases were reviewed and discussed in order to develop a consensus how such cases might be categorised with respect to cause of death and COPD-relatedness. From these discussions, the CEC developed a series of principles of operation that could be applied to categorise the cause of death (see Appendix A in online data supplement available at http://thorax.bmj.com/ supplemental). During subsequent meetings of the CEC, principles were added or expanded for cases that did not seem to be otherwise addressed.

The CEC had an in-person meeting three to four times per year. During each meeting, lasting 2-3 days, approximately 75150 cases were reviewed. The study sponsor (GlaxoSmithKline, Greenford, UK and Research Triangle Park, North Carolina, USA) organised the meetings and provided staff support but did not have a discussant or voting member at the meetings. The three CEC physicians, blinded to treatment assignment and interim study outcomes, independently reviewed each case to assign a cause of death and complete and sign an Endpoint Adjudication Form. At the meeting the three independent opinions were reconciled and a final form was filled in and signed. If the cause of death was not unanimous, the case was further reviewed and discussed with the aim of developing a consensus. The committee charter provided that, in the event of a non-unanimous decision, the cause of death would be established by the study chair. In practice, however, a unanimous consensus was reached in all cases reviewed.

If the CEC concluded that additional medical information might be obtained to assist in assigning a more accurate cause of death, the case was referred back to the study site to obtain the additional information. If additional information was provided, the case was re-reviewed at a subsequent meeting. If the study site affirmed that the additional requested information could not be obtained, the case was referred back to the CEC for a second review. In this circumstance, the CEC attempted to assign a cause of death based on the best available evidence; if this was not possible, the cause of death was classified as unknown.

\section{Categorisation of cause of death and attribution to COPD}

Cause-specific mortality was first attributed to a specific cause of death and then grouped by a general pathophysiological category (cardiovascular, respiratory, cancer or other). If the cause of death could not be determined from the available evidence it was classified as unknown.

In each case a second determination was made as to whether the death was considered related to COPD using a hierarchical scale (no, unlikely, possibly, probably, yes). If the evidence was not sufficient to make this determination, the relationship to COPD was classified as unknown.

\section{Principles used in attributing cause of death to COPD}

A major aim of the CEC was to determine which deaths were caused by COPD or, if not specifically caused by COPD, were related to it. There have been no previously published definitions of causation of death from COPD or whether a death from another primary cause could be considered related to COPD. The CEC therefore used systematic definitions that were prepared from a series of hypothetical scenarios before the adjudication of cases. The general principle used was that a death was attributed to COPD if the final illness was precipitated by a COPD exacerbation, regardless of subsequent fatal events such as pneumonia, sepsis or multiorgan system failure. ${ }^{9}$ In practice, this meant that the patient had increasing cough, sputum or dyspnoea for which they received treatment at the onset of the terminal illness. If pneumonia was present, as evidenced by an infiltrate on the chest radiograph at the time of presentation, the cause of death was attributed to pneumonia. If pneumonia occurred after the onset of a terminal COPD exacerbation, the case was categorised as a COPD death. In some cases the medical record indicated that patients with advanced COPD (evidenced by debilitation, poor nutrition and hypoxaemia) were placed into palliative care before death. These cases were also categorised as COPD deaths.

The CEC considered a death to be related to COPD if they judged that the terminal illness would probably be non-fatal if COPD was not present. For example, most patients dying of pneumonia and respiratory failure were considered to have COPD-related deaths. In contrast, patients dying of myocardial infarction or incurable cancer were not considered COPDrelated even if the incidence of those disorders is increased in patients with COPD.

\section{Reliability of procedures}

In order to ascertain the reliability of the mortality attributions by the CEC, an analysis was conducted in a sample of 100 cases which had been adjudicated twice The committee members were aware that cases were being submitted for second review, but were not aware which cases were being submitted a second time.

\section{Comparability of determining cause of death with other} methods

In order to determine whether the CEC adjudication process provided different information from other methods of 
Table 1 Classification of cause-specific mortality $(n=911)$

\begin{tabular}{lclr}
\hline System & $\%$ & Subcategory & $\%$ \\
\hline Cardiovascular & 26 & Congestive heart failure & 3 \\
& & Myocardial infarction & 3 \\
& & Stroke & 4 \\
& & Sudden death & 16 \\
Respiratory & 35 & COPD & 27 \\
& & Pneumonia & 8 \\
Cancer & 21 & Other & $<1$ \\
Other cause & 10 & Other & 14 \\
Unknown cause & 8 & & 7 \\
\hline
\end{tabular}

ascertaining cause of death, the CEC adjudication was compared with the attribution of the site investigator in all 911 cases.

\section{Statistical methods}

The process for sampling cases for a second masked review was ongoing throughout the course of the trial. A random sample of 25 cases was drawn from the first 252 deaths, a random sample of an additional 50 cases was drawn from the 540 deaths which had been adjudicated by March 2005, and a final sample of 25 cases from the total 911 deaths. Re-sampled cases were mixed into the cases for each subsequent meeting in a masked fashion.

To assess the extent to which a given adjudication is reliable, the kappa $(\kappa)$ statistic was calculated..$^{10}$ This is a measure of the agreement in excess of the amount of agreement that we would expect by chance. It has a maximum of 1.00 when agreement is perfect and a value of 0 indicates no agreement better than chance. All analyses were performed using Version 8.02 of the SAS software package (Carey, North Carolina, USA).

\section{RESULTS}

A total of 911 deaths were reviewed during 10 meetings each lasting 2-3 days. After independent review and discussion, a consensus was reached on cause of death and relatedness to COPD in all cases. Twenty percent of cases that were reviewed were sent back for additional information. Among the cases where an adjudication was made, the cause of death was considered unknown in only $8 \%$ and the relationship to COPD could not be determined in $9 \%$.

The CEC commonly ascertained causes of death that were different from the free text entered by the site investigator on the case report form. Among the 911 cases, the committee adjudication was equivalent to the primary cause of death listed by the site investigator in 52\% of cases. The committee adjudication for cause of death was listed in either the primary or secondary cause of death provided by the site investigator in $67 \%$ of cases.

Of the 100 cases that were submitted for a second blinded review, an identical adjudication of cause-specific mortality was reached in $83 \%$ (95\% CI $76 \%$ to $90 \%$ ). The $\kappa$ statistic for cause of

\begin{tabular}{lc} 
Table 2 Relationship of death to COPD \\
\hline Classification & $\%$ \\
\hline Yes (definite) & 38 \\
Probably & 2 \\
Possibly & 1 \\
Unlikely & $<1$ \\
No (not related) & 50 \\
Unknown & 9 \\
\hline
\end{tabular}

death was 0.80 (95\% CI 0.71 to 0.89$)$. The discrepant adjudications in the first and second review are listed in Appendices B and C in the online data supplement available at http://thorax.bmj.com/supplemental. Whether the death was related to COPD was also adjudicated on two separate occasions in the same cases. Among these cases, COPD-relatedness was consistent in $84 \%$ (95\% CI $77 \%$ to $91 \%$ ). The $\kappa$ statistic for COPD-relatedness was 0.73 (95\% CI 0.62 to 0.84$)$. The discrepant adjudications are listed in Appendices $\mathrm{B}$ and $\mathrm{C}$ of the online data supplement.

The adjudicated causes of death are shown in table 1. The most common cause of death was respiratory (35\%) with approximately $75 \%$ occurring after a COPD exacerbation. Cardiovascular deaths occurred in $26 \%$ of cases, with the most common cause being sudden death. Cancer caused $21 \%$ of deaths, about two-thirds being due to lung cancer. Overall, $40 \%$ of the deaths were judged to be definitely or probably related to COPD; that is, it was judged that the patient would probably have survived the terminal illness if COPD was not present (table 2).

\section{DISCUSSION}

In this paper we describe the operations of a CEC that had the task of attributing cause of death in patients with COPD and adjudicating whether the deaths were related to COPD in a large multinational clinical trial.

Clinical Endpoint Committees are routinely used in cardiovascular clinical trials, and several reports have concluded that they can provide independent, systematic, standardised adjudication of outcome events. ${ }^{11-16}$ Outcomes of clinical trials may differ substantially when events are independently and systematically adjudicated rather than relying on local site investigators or death certificates. ${ }^{17-21}$ In contrast to endpoints in coronary artery disease, COPD provides a particular challenge because there are no accepted definitions of when death is caused by or related to COPD. Many patients with COPD die from other causes, particularly cardiovascular disease, pneumonia and lung cancer. ${ }^{22-24}$ This is because patients with COPD suffer from other smoking-related comorbidities such as coronary artery disease, they are vulnerable to fatal outcomes from other illnesses such as pneumonia, and also because cause of death in COPD may be inaccurately or inconsistently attributed. $^{25-28}$

All-cause mortality is an objective and unbiased outcome measure for clinical trials. Cause-specific mortality, although limited by reduced power and potential for biased ascertainment, can provide information about subtle beneficial or adverse treatment effects that are not of sufficient magnitude to alter all-cause death rates. Moreover, the systematic review and attribution of cause of death in well characterised cohorts of patients with COPD participating in a clinical trial can extend our understanding of the health impact, epidemiology and natural history of COPD. Because there are no generally agreed definitions of what constitutes death from COPD or death related to COPD, the CEC had to develop a set of definitions and working principles to guide their deliberations. We emphasise that these definitions were developed in the context of a clinical trial evaluating maintenance treatment of COPD and would not necessarily be appropriate for investigations that were targeting other diseases or treatment approaches.

We also evaluated whether the CEC could provide reproducible adjudications by blindly reviewing a sample of cases on two separate occasions. We found that the reliability of the CEC adjudications were reasonably good with identical adjudications in $85 \%$ of cases. We are not aware of similar measures of reproducibility of adjudication by mortality review committees in clinical trials, but our results are comparable to the 
reproducibility of adjudication of causes of perinatal mortality. ${ }^{29}$ Two issues were often problematic for the committee. First, it was often difficult to distinguish between pneumonia and COPD exacerbation as the presenting terminal illness. Second, it was difficult to decide when an unattended death should be called sudden death vs unknown cause.

We also evaluated whether the CEC, using an independent review and consensus approach, could provide information that was different from using site investigator specification of cause of death. The CEC ascertained different causes of death from the site investigator's primary or secondary cause of death in about one-third of cases. Although some of the site investigators also served as treating physicians for trial participants, the attribution of cause of death was probably not as consistently applied as the CEC adjudications. In many cases the site investigators specified the cause of death based on the primary cause listed on the death certificate or from the terminal event, for example, "cardiac arrest or cardiorespiratory arrest". Many of the cases that the CEC classified as sudden death were attributed to myocardial infarction. Thus, different conclusions regarding cause-specific mortality and the effect of treatment on cause-specific mortality would be reached using CEC adjudication versus site investigator adjudication. In general, dependence upon site investigator diagnoses would tend to increase the attribution of death to cardiovascular causes and diminish the attribution to respiratory causes.

The TORCH trial is the first large international clinical trial focusing on COPD mortality. Besides the lack of well established precedence for operation of a CEC for COPD trials, the large number of independent study sites and the multiplicity of countries added challenges to attribution of cause of death to COPD. Linguistic, cultural and legal barriers may have affected the ways that deaths are reported and documented. In several jurisdictions, death certificates were not legally available to investigators or the cause of death was withheld from the public record. The planning for such studies needs to take into account the substantial effort and infrastructure required to obtain, review for completeness, collate, translate and distribute the medical information required for central review.

As expected, the causes of death in this COPD population had a larger proportion attributable to respiratory illness than is found in the general population of industrialised countries where cardiovascular and neoplastic causes of death far exceed respiratory illnesses. Several studies have examined causes of death specifically in COPD and have found a lower proportion of deaths from respiratory conditions. The Lung Health Study mortality review panel adjudicated causes of death in 149 patients with mild to moderate COPD. ${ }^{7}$ Lung cancer was the most common cause of death, occurring in 33\% of patients. Cardiovascular disease, comprising both cerebrovascular accidents and coronary artery disease, occurred in 25\% of decedents. Respiratory causes of death were uncommon in this group. In Lung Health Study 2, among 34 deaths adjudicated by a mortality review panel, the most commonly reported cause of death was lung cancer..$^{30}$ Both Lung Health Studies had patients with milder lung disease than TORCH, so it would be expected that fewer patients would have succumbed to respiratory diseases. Hansell and colleagues examined death certificate causes of mortality in decedents in England and Wales who had COPD or a related condition listed as primary or a contributing condition on their certificate. They found that cardiovascular disease accounted for $25 \%$ of deaths, neoplasm $7 \%$ of deaths and respiratory conditions accounted for only $4 \%$ of deaths. ${ }^{31}$ They suggested that deaths due to COPD were vastly underreported on death certificates. In a study of 215 patients using chronic oxygen, Zielinski et $a l^{25}$ found that $38 \%$ died of respiratory failure, $13 \%$ of cor pulmonale, $11 \%$ of pneumonia,
$10 \%$ pulmonary embolism, $8 \%$ cardiac arrhythmia and 7\% died of lung cancer. Thus, in this group of patients with more severe COPD, deaths from respiratory causes were more common than in the TORCH study.

One of the interesting findings of this study was the large proportion of patients who had sudden death (16\%) and the low proportion of patients with documented acute myocardial infarction (3\%). Sudden deaths are usually classified as cardiovascular deaths and are attributed to arrhythmias in the setting of coronary artery disease. It was therefore surprising that so few patients had documented myocardial infarctions. This finding raises the speculation that many of these deaths might have been attributable to acute respiratory failure as a precipitating cause, a phenomenon that has been well described in asthma but not in COPD. ${ }^{32}$ It has previously been found that $47 \%$ of patients who die after recovering from an episode of acute respiratory failure ultimately die of sudden death, suggesting that this may have accounted for this finding. ${ }^{33}$ We therefore suggest that future studies of COPD mortality should consider sudden death as a separate entity rather than one necessarily linked to a cardiovascular cause.

One of the most difficult decisions made by the committee, and the cause of several of the disparate classifications on readjudication, was the distinction between COPD exacerbations and pneumonia. Although we had clear definitions to separate the two based on whether an infiltrate was present on the initial presenting chest radiograph, there were some circumstances where the clinical and official reading of the initial chest radiograph was different or where an infiltrate was noted very shortly after the onset of symptoms but not on the initial radiograph. Moreover, in all but one case, deaths from pneumonia were judged to be COPD-related and the symptoms of cough, sputum and dyspnoea were virtually always present in the setting of pneumonia. Thus, the distinction between death from COPD exacerbation complicated by pneumonia and pneumonia leading to a COPD exacerbation was sometimes unclear. We therefore suggest that future research evaluating COPD mortality should subclassify such events as "COPD exacerbations accompanied by pneumonia" and "COPD exacerbations without pneumonia".

In conclusion, a CEC can provide systematic and reliable attributions of death in clinical trials of COPD. The key elements of operation of such a committee include preliminary development of principles of operation and working definitions, in-person meetings for discussion of cases and substantial infrastructure for acquisition of medical information. Based on our experience, we would recommend modifications for future clinical trials of COPD, particularly the classification of COPD exacerbations that occur in the setting of pneumonia, the classification of sudden death as a cardiovascular event, and attention to training of investigators and site personnel about the operational requirements of such a committee and the substantial effort required to obtain, review, translate and collate relevant medical records.

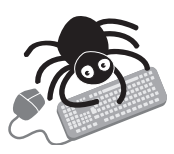

Appendices A, B and C are available online at http:// thorax.bmj.com/supplemental.
Authors' affiliations
Lorcan P McGarvey, The Queen's University of Belfast, Belfast, UK Matthias John, Respiratory Medicine, Barmer Ostseeklinik, Prerow, Germany
Julie A Anderson, GlaxoSmithKline, Greenford, UK
Michael Zvarich, GlazoSmithKline, Research Triangle Park, North 
Carolina, USA

Robert A Wise, Johns Hopkins University, Baltimore, Maryland, USA

Funding for the study described in this article was provided by GlaxoSmithKline.

Competing interests: $L M C G, M J$ and RAW are paid consultants to GlaxoSmithKline. JAA and MZ are employees of GlaxoSmithKline. RAW's arrangements are managed by the Johns Hopkins University in accordance with its conflict of interest policies.

$L M C G$ and $M J$ were equal contributors to this study and are considered as joint first authors.

\section{REFERENCES}

1 NHLBI. NHLBI morbidity and mortality chartbook, 2004.Available at http:// www.nhlbi.nih.gov/resources/docs/cht-book.htm (accessed 13 February 2005).

2 Wise RA. Changing smoking patterns and mortality from chronic obstructive pulmonary disease. Prev Med 1997;26:418-21.

3 Murray CJ, Lopez AD. Alternative projections of mortality and disability by cause 1990-2020: Global Burden of Disease Study. Lancet 1997;349:1498-504.

4 Nocturnal Oxygen Therapy Trial Group. Continuous or nocturnal oxygen therapy in hypoxemic chronic obstructive lung disease: a clinical trial. Ann Intern Med 1980;93:391-8.

5 Anon. Long term domiciliary oxygen therapy in chronic hypoxic cor pulmonale complicating chronic bronchitis and emphysema: report of the Medical Research Council Working Party. Lancet 1981;1:681-6.

6 Vestbo J, TORCH Study Group. The TORCH (towards a revolution in COPD health) survival study protocol. Eur Respir J 2004;24:206-10.

7 Anthonisen NR, Connett JE, Kiley JP, et al. Effects of smoking intervention and the use of an inhaled anticholinergic bronchodilator on the rate of decline of FEV 1 . The Lung Health Study. JAMA 1994;272:1497-505.

8 Anthonisen NR, Skeans MA, Wise RA, et al. The effects of smoking cessation intervention on 14. 5-year mortality: a randomized clinical trial, Ann Intern Med 2005; 142:233-9.

9 Afessa B, Morales IJ, Scanlon PD, et al. Prognostic factors, clinical course, and hospital outcome of patients with chronic obstructive pulmonary disease admitted to an intensive care unit for acute respiratory failure. Crit Care Med 2002;30:1610-5.

10 Fleiss J. Statistical methods for rates and proportions, 2nd ed. New York: Wiley, 1981

11 Mahaffey KW, Roe MT, Dyke CK, et al. Misreporting of myocardial infarction end points: results of adjudication by a central clinical events committee in the PARAGON-B trial. Second Platelet llb/llla Antagonist for the Reduction of Acute Coronary Syndrome Events in a Global Organization Network Trial. Am Heart J 2002;143:242-8

12 Mahaffey KW, Harrington RA, Akkerhuis M, for the PURSUIT Investigators, et al. Disagreements between central clinical events committee and site investigator assessments of myocardial infarction endpoints in an international clinical trial: review of the PURSUIT study. Curr Control Trials Cardiovasc Med $2001 ; 2: 187-94$
13 Mahaffey KW, Harrington RA, Akkerhuis M, for the PURSUIT Investigators, et al. Systematic adjudication of myocardial infarction end-points in an international clinical trial. Curr Control Trials Cardiovasc Med 2001;2:180-6.

14 Naslund U, Grip L, Fischer-Hansen J, et al. The impact of an end-point committee in a large multicentre, randomized, placebo-controlled clinical trial: results with and without the end-point committee's final decision on end-points. Eur Heart J 1999;20:771-7.

15 Heagerty A, Deverly A, Palmer C, et al. The role of the critical event committee in a major cardiovascular outcome study. Blood Press 2002;11:339-44.

16 Laver MS, Blackstone EH, Young JB, et al. Cause of death in clinical research: time for a reassessment? J Am Coll Cardiol 1999;34:618-20.

17 Kestle J, Milner R, Drake D. An assessment of observer bias in the shunt design trial. Pediatr Neurosurg 1999;30:57-61.

18 Temple R, Pledger GW. The FDA's critique of the anturane reinfarction trial. N Engl J Med 1980;303:1488-92

19 Epstein AE, Carlson MD, Fogoros RN, et al. Classification of death in antiarrhythmia trials. J Am Coll Cardiol 1996;27:433-42.

20 Heckbert SR, Kooperberg C, Safford MM, et al. Comparison of self-report, hospital discharge codes, and adjudication of cardiovascular events in the Women's Health Initiative. Am J Epidemiol 2004;160:1152-8.

21 Moussa MA, Shafie MZ, Khogali MM, et al. Reliability of death certificate diagnoses. J Clin Epidemiol 1990;43:1285-95.

22 Tockman MS, Anthonisen NR, Wright EC, et al. Airways obstruction and the risk for lung cancer. Ann Intern Med 1987; 106:512-8.

23 Skillrud DM, Offord KP, Miller RD. The higher risk of lung cancer in chronic obstructive pulmonary disease. Ann Intern Med 1986;105:503-7.

24 Lange $\mathbf{P}$, Nyboe J, Appleyard $M$, et al. Spirometric finding and mortality in never-smokers. J Clin Epidemiol 1990;43:867-73.

25 Zielinski J, MacNee W. Wedzicha J, et al. Causes of death in patients with COPD and chronic respiratory failure. Monaldi Arch Chest Dis 1997;52:43-7.

26 Janssens JP, Herrmann F, MacGee W, et al. Cause of death in older patients with anatomo-pathological evidence of chronic bronchitis or emphysema: a case-control study based on autopsy findings. J Am Geriatr Soc 2001;49:571-6.

27 Higgins MW, Keller JB. Trends in COPD morbidity and mortality in Tecumseh, Michigan. Am Rev Respir Dis 1989;140:S42-8.

28 Vilkman S, Keistinen T, Tuuponen T, et al. Survival and cause of death among elderly chronic obstructive pulmonary disease patients after first admission to hospital. Respiration 1997;64:281-4.

29 Rajmil L, Plasencia A, Borrell C. Reproducibility of the use of classifications of causes of death in the context of inquiries in perinatal mortality (English abstract). An Esp Pediatr 1993;39:398-404.

30 Lung Health Study Research Group. Effect of inhaled triamcinolone on the decline in pulmonary function in chronic obstructive pulmonary disease. N Engl J Med 2000;343:1902-9.

31 Hansell AL, Walk JA, Soriano JB. What do chronic obstructive pulmonary disease patients die from? A multiple cause coding analysis. Eur Respir J 2003;22:809-14.

32 Wren C, O'Sullivan JJ, Wright C. Sudden death in children and adolescents. Heart 2000;83:410-3.

33 Shachor Y, Liberman D, Tamir A, et al. Long-term survival of patients with chronic obstructive pulmonary disease following mechanical ventilation. Isr J Med Sci 1989;25:617-9

Take advantage of BMJ Journals' remarkable catalogue of titles with Related Collections

No busy professional has time to browse through all pertinent journals to find relevant articles, but with Related Collections you no longer have to. Follow the "Related Collections" link from any article and use the "Show Collections from other Journals" to expand your search across all BMJ Journals. Or simply follow the "Browse by topic" link on the home page. By setting up your own collections and receiving email alerts every time an article is added to your chosen area, you can build up your own significant body of knowledge. 